UDC 517.54

\author{
D. F. Abzalilov, E. A. Shirokova
}

\title{
THE APPROXIMATE CONFORMAL MAPPING ONTO MULTIPLY CONNECTED DOMAINS
}

\begin{abstract}
The method of boundary curve reparametrization is generalized to the case of multiply connected domains. We construct the approximate analytical conformal mapping of the unit disk with $N$ circular slits or an annulus with $(N-1)$ circular slits onto an arbitrary $(N+1)$ multiply connected finite domain with a smooth boundary. The method is based on the solution of the Fredholm equation. This solution is reduced to the solution of a linear system with unknown Fourier coefficients. The approximate mapping function has the form of a set of Laurent polynomials in the set of annular regions The method is easily computable.
\end{abstract}

Key words: conformal mapping, multiply connected domain, Fredholm integral equation

2010 Mathematical Subject Classification: 30C20, 30C30, $45 B 05$

1. Introduction. Conformal mappings by analytical functions of complex variable play an important role in solving many problems of mechanics and mathematics, particulary in the case of plane potential fields and the Laplace equation [1]. The conformal mapping of circular domains (a disk with circular slits or an annulus with circular slits) onto a multiply connected domain with a complex boundary can be applied to solving plane boundary value problems for the corresponding domains with the help of Schottky-Klein prime functions known for circular domains [2]. Computer progress has stimulated appearance of many numerical methods for constructing conformal mapping [3]. However, all these methods are rather time-consuming. For example, the widely used Wegmann numerical method is based on solving the Riemann-Hilbert problem and involves iteration processes $[4,5]$.

(C) Petrozavodsk State University, 2019 
There are several types of canonical regions for conformal mappings [6]. The five types of canonical slit regions are a disk with concentric circular slits, an annulus with concentric circular slits, unbounded circular slit regions, unbounded radial slit regions, and unbounded parallel slit regions. Recenaly a few approaches to map bounded and unbounded multiply connected regions onto these five canonical regions by reformulating the mapping problems as Riemann-Hilbert problems have been proposed; these problems are solved by means of boundary integral equations with the generalized Neumann kernel [7-13]. The integral equations were discretized by the Nyström method with the trapezoidal rule used to obtain linear systems. The right-hand side of the integral equation in $[7,8]$ involves integral with the cotangent singularity which is approximated by Wittich's method.

Here we present a new method of the approximate conformal mapping of a unit disk with circular slits or an annulus with circular slits onto a multiply connected domain with a smooth boundary. We apply the integral equation with the Neumann kernel obtained from the necessary and sufficient condition for a function defined at the points of a smooth contour to be the boundary values of some function analytical in the correspondent domain. The theory of this method for simply connected domains has been developed in $[14,15]$, the generalization to the case of a doubly connected domain and the relative numerical implementation can be found in [16]. Here we generalize the method to the case of multiply connected domains whose internal borders are images of circular arcs and an inner circle. The method is based on the boundary curves reparametrization. We do not search for corrections of boundary parametrization with linearization of this process $[17,18]$. The advantages of our method are the following: the method does not use any auxiliary constructions (triangulation, circle packing, domain decomposition) or conformal mappings (the zipper algorithm, the Schwarz-Christoffel mapping), it does not use the accessory solutions of boundary value problems (the conjugate function method, the Wegmann method), it does not use iterations. We do not apply the collocation method or the Nyström's method for solution of the integral equation.

The solution of integral equations in our method is reduced to the solution of an infinite linear system. We obtain an approximate mapping by solving the finite system with a truncated matrix. The smooth solution of the problem has the form of a Laurent polynomial in each annular 
region. This form allows us to apply the differentiation techniques for solutions of some physical problems in the corresponding domains. We must note that our method is applicable only for the domains with smooth boundary components or the boundary components with vertices of the angles more than $\pi$. The cases of the angles less than $\pi$ bring to kernels with strong singularities.

2. Approximate conformal mapping of the circular domains of two types onto a multiply connected domain by means of the boundary reparametrization. Consider a finite multiply connected domain $D_{z}$ bounded by the outer simple smooth curve $L_{0}$ and the inner simple smooth curves $L_{s}$ given by the equations

$$
L_{s}=\left\{z=z_{s}(t), \quad z_{s}(0)=z_{s}(2 \pi), \quad t \in[0,2 \pi]\right\}, s=\overline{0, N} .
$$

Also, assume that complex representations of the boundary curves $L_{s}$ are as follows:

$$
z_{s}(t)=\sum_{k=-m_{s}}^{n_{s}} d_{k s} e^{i k t}, \quad t \in[0,2 \pi], \quad s=\overline{0, N} .
$$

The parametrization traces the domain $D_{z}$ along $L_{0}$ counterclockwise and along the inner contours $L_{s}, s=\overline{1, N}$, clockwise.

Definition 1. We call the unit disk $|\zeta|<1$ with $N$ circular slits $\zeta=R_{j} e^{i \theta}, \quad \theta_{1 j}<\theta<\theta_{2 j}$, with a constant $R_{j}<1, \quad j=\overline{1, N}$, an $(N+1)$-connected circular domain of the first type.

Definition 2. We call the annulus $r<|\zeta|<1$, with $(N-1)$ circular slits $\zeta=R_{j} e^{i \theta}, \quad \theta_{1 j}<\theta<\theta_{2 j}$, with a constant $R_{j} \in(r, 1), j=\overline{1, N-1}$, an $(N+1)$-connected circular domain of the second type.

Existence theorems for the approximate conformal mappings of given $(N+1)$ multiply connected finite domain with a smooth boundary onto a circular domain of type I and of type II are well-known (see, e.g., $[6,13,19]$ ).

Theorem 1. There exists an $(N+1)$-connected circular domain $D_{\zeta}$ of the first type and there exists a regular function $z=f(\zeta)$ in this domain, such that the function $f(\zeta)$ maps conformally the domain $D_{\zeta}$ onto the given $(N+1)$-connected domain $D_{z}$ with smooth boundary components. The map is unique under the following conditions: $f(0)=Z_{0}, f(1)=Z_{1}$, $Z_{0} \in D_{z}, Z_{1} \in L_{0}$. The corresponding approximate function can be constructed in the form of a Laurent polynomial. 
Proof. We assume that $0 \in D_{z}$ and $Z_{0}=0$, without loss of generality. The proof is constructive. We construct the conformal map of the circular domain of the first type onto the domain $D_{z}$ by reparametrization of the given boundary representations. So, we search for the function $t_{0}(\theta)$, $\theta \in[0,2 \pi]$, and for $t_{s}(\theta), s=\overline{1, N}, \theta \in\left[\theta_{1 s}, \theta_{2 s}\right]$, such that the values $z_{s}\left(t_{s}(\theta)\right), s=\overline{0, N}$, are the boundary values of an analytic function in the corresponding circular domain. The parameters $R_{s}, \theta_{1 s}, \theta_{2 s}$, are also unknown and will be found within the solution process.

Let us consider the analytic in the domain $D_{z}$ function $\zeta(z)$ that maps conformally the domain $D_{z}$ onto $D_{\zeta}$ with the correspondence $\zeta(0)=0$ and the analytic in $D_{z}$ function $\log \frac{z}{\zeta}$. According to [14], the necessary and sufficient condition for $\log \frac{z}{\zeta}$ to be analytic in $D_{z}$ is the boundary relation

$$
\log \frac{z_{s}(t)}{R_{s} e^{i \theta_{s}(t)}}=\sum_{\sigma=0}^{N} \frac{1}{\pi i} \int_{0}^{2 \pi} \log \left(\frac{z_{\sigma}(\tau)}{R_{\sigma} e^{i \theta_{\sigma}(\tau)}}\right) \frac{z_{\sigma}^{\prime}(\tau)}{z_{\sigma}(\tau)-z_{s}(t)} d \tau,
$$

where $t \in[0,2 \pi], s=\overline{0, N}, R_{0}=1$.

We introduce the functions $q_{s}(t)=\arg z_{s}(t)-\theta_{s}(t), s=\overline{0, N}$, where $\theta_{s}(t)$ is the polar angle of the image of the point of $z_{s}(t)$ and separate the imaginary part of both sides of equation (1):

$$
\begin{aligned}
q_{s}(t)= & \sum_{\sigma=0}^{N} \frac{1}{\pi} \int_{0}^{2 \pi} q_{\sigma}(\tau)\left(\arg \left[z_{\sigma}(\tau)-z_{s}(t)\right]\right)_{\tau}^{\prime} d \tau- \\
& -\sum_{\sigma=0}^{N} \frac{1}{\pi} \int_{0}^{2 \pi} \log \frac{\left|z_{\sigma}(\tau)\right|}{R_{\sigma}}\left(\log \left|z_{\sigma}(\tau)-z_{s}(t)\right|\right)_{\tau}^{\prime} d \tau, \quad s=\overline{0, N} .
\end{aligned}
$$

After differentiating this relation and integrating the result by parts, we obtain the following relations on the functions $q_{s}^{\prime}(t)$ :

$$
\begin{aligned}
& q_{s}^{\prime}(t)=\sum_{\sigma=0}^{N} \frac{1}{\pi} \int_{0}^{2 \pi} q_{\sigma}^{\prime}(\tau) K_{\sigma s}(\tau, t) d \tau+p_{s}(t), \quad s=\overline{0, N}, \\
& K_{\sigma s}(\tau, t)=-\left(\arg \left[z_{\sigma}(\tau)-z_{s}(t)\right]\right)_{t}^{\prime}, \\
& p_{s}(t)=\sum_{\sigma=0}^{N} \frac{1}{\pi} \int_{0}^{2 \pi}\left[\log \left|z_{\sigma}(\tau)\right|\right]^{\prime}\left(\log \left|z_{\sigma}(\tau)-z_{s}(t)\right|\right)_{t}^{\prime} d \tau .
\end{aligned}
$$


The kernel $\left(\log \left|z_{\sigma}(\tau)-z_{s}(t)\right|\right)_{t}^{\prime}$ has a singularity in the form of $\cot \frac{\tau-t}{2}$ for $\sigma=s$ :

$$
\begin{gathered}
\left(\log \left|z_{s}(\tau)-z_{s}(t)\right|\right)_{t}^{\prime}=\operatorname{Re}\left(\log \sum_{k=-m_{s}}^{n_{s}} d_{k s}\left[e^{i k \tau}-e^{i k t}\right]\right)_{t}^{\prime}= \\
=-\frac{1}{2} \cot \frac{\tau-t}{2}+\left(\log \mid \sum_{k=1}^{n_{s}} d_{k s} e^{i k t} \sum_{l=0}^{k-1} e^{i l(\tau-t)}-\right. \\
\left.-\sum_{k=1}^{m_{s}} d_{(-k) s} e^{-i k \tau} \sum_{l=0}^{k-1} e^{i l(\tau-t)} \mid\right)_{t}^{\prime}
\end{gathered}
$$

The Cauchy principal value integral

$$
\frac{1}{\pi} \int_{0}^{2 \pi}\left[\log \left|z_{\sigma}(\tau)\right|\right]^{\prime} \cot \frac{\tau-t}{2} d \tau
$$

can be calculated via the Hilbert formula [20] as in [16].

We search for the approximate solution of (2) in the form of the Fourier polynomial

$$
q_{s}^{\prime}(t)=\sum_{j=1}^{M} \alpha_{j s} \cos j t+\beta_{j s} \sin j t, t \in[0,2 \pi] .
$$

Existence of exact solution to equation (2) and convergence of the approximate solution to the exact one as $M \rightarrow \infty$ were proved in [15] for simply connected domains. This proof can be applied to the case of multiply connected domains if we replace the corresponding space $l^{2}$ by the space $l^{2} \times l^{2} \times \ldots \times l^{2}$.

Now the integral Fredholm equations of the second kind (2) can be reduced to the linear system for the Fourier coefficients $\alpha_{j s}$ and $\beta_{j s}$ :

$$
\left(\begin{array}{cccccc}
A_{00} & B_{00} & A_{01} & B_{01} & \cdots & B_{0 N} \\
C_{00} & D_{00} & C_{01} & D_{01} & \cdots & D_{0 N} \\
A_{10} & B_{10} & A_{11} & B_{11} & \cdots & B_{1 N} \\
C_{10} & D_{10} & C_{11} & D_{11} & \cdots & D_{1 N} \\
\vdots & \vdots & \vdots & \vdots & \ddots & \vdots \\
C_{N 0} & D_{N 0} & C_{N 1} & D_{N 1} & \cdots & D_{N N}
\end{array}\right)\left(\begin{array}{c}
\alpha_{0} \\
\beta_{0} \\
\alpha_{1} \\
\beta_{1} \\
\vdots \\
\beta_{N}
\end{array}\right)=\left(\begin{array}{c}
a_{0} \\
b_{0} \\
a_{1} \\
b_{1} \\
\vdots \\
b_{N}
\end{array}\right)
$$


where $\alpha_{s}=\left(\alpha_{0 s}, \ldots, \alpha_{M s}\right)^{T}, \quad \beta_{s}=\left(\beta_{0 s}, \ldots, \beta_{M s}\right)^{T}$. The vectors $a_{s}=\left(a_{0 s}, \ldots, a_{M s}\right)^{T}, b_{s}=\left(b_{0 s}, \ldots, b_{M s}\right)^{T}$ on the right-hand side of the system consist of the elements

$a_{j s}=\frac{1}{\pi} \int_{0}^{2 \pi} p_{s}(t) \cos j t d t, \quad b_{j s}=\frac{1}{\pi} \int_{0}^{2 \pi} p_{s}(t) \sin j t d t, \quad j=\overline{1, M}, s=\overline{0, N}$.

The block matrices $A_{\sigma s}, B_{\sigma s}, C_{\sigma s}, D_{\sigma s}$ of size $M \times M$ consist of the elements

$$
\begin{array}{ll}
A_{\sigma s j k}=\delta_{\sigma s} \delta_{j k}-\frac{1}{\pi^{2}} \int_{0}^{2 \pi} \cos k \tau d \tau \int_{0}^{2 \pi} K_{\sigma s}(\tau, t) \cos j t d t, & \sigma=\overline{0, N}, \\
B_{\sigma s j k}=-\frac{1}{\pi^{2}} \int_{0}^{2 \pi} \sin k \tau d \tau \int_{0}^{2 \pi} K_{\sigma s}(\tau, t) \cos j t d t, & s=\overline{0, N} \\
C_{\sigma s j k}=-\frac{1}{\pi^{2}} \int_{0}^{2 \pi} \cos k \tau d \tau \int_{0}^{2 \pi} K_{\sigma s}(\tau, t) \sin j t d t, & j=\overline{1, M}, \\
D_{\sigma s j k}=\delta_{\sigma s} \delta_{j k}-\frac{1}{\pi^{2}} \int_{0}^{2 \pi} \sin k \tau d \tau \int_{0}^{2 \pi} K_{\sigma s}(\tau, t) \sin j t d t, & k=\overline{1, M},
\end{array}
$$

where $\delta_{\sigma s}$ and $\delta_{j k}$ are the Kronecker delta functions.

The functions $q_{s}(t), s=\overline{0, N}$, can be restored via their derivatives (3) with an arbitrary constant summand

$$
q_{s}(t)=q_{0 s}+\tilde{q}_{s}(t), \quad \tilde{q}_{s}(t)=\sum_{j=1}^{M} \frac{\alpha_{j s}}{j} \sin j t-\frac{\beta_{j s}}{j} \cos j t, t \in[0,2 \pi] .
$$

We choose the constant summand $q_{00}$ in accordance with the condition $f(1)=Z_{1}$ in the following way. We find the value of the parameter $\hat{t}$ such that $z_{0}(\hat{t})=Z_{1}$. Now $q_{00}=\arg \left(Z_{1}\right)-\tilde{q}_{0}(\hat{t})$.

We obtain the values of the other constant summands $q_{0 s}, s=\overline{1, N}$, and also the values of $R_{s}, s=\overline{1, N}$, in the following way. We take $N$ points in each of the $N$ finite components of the set complement of $D_{z}$. Let us denote these points $z_{j}^{*}, j=\overline{1, N}$. The function $\log \left(z_{s}(t) / R_{s} e^{i \theta_{s}(t)}\right)$, $s=\overline{0, N}$, is the boundary value of the analytical in $D_{z}$ function, so the Cauchy integral with the corresponding density along the boundary of $D_{z}$ 
vanishes at the points $z_{j}^{*}, j=\overline{1, N}$. Therefore, we have the linear complex system

$$
\sum_{\sigma=0}^{N} \int_{0}^{2 \pi}\left(i q_{0 s}-\log R_{s}+\log \left|z_{s}(\tau)\right|+i \tilde{q}_{s}(\tau)\right)\left[\log \left(z_{\sigma}(\tau)-z_{j}^{*}\right)\right]_{\tau}^{\prime} d \tau=0,
$$

$j=\overline{1, N}$, with the unknown real $q_{0 s}$ and $\log R_{s}, s=\overline{1, N}$.

We restore the values of $\theta_{1 j}$ and $\theta_{2 j}, j=\overline{1, N}$, after we have restored $q_{0 j}$. Indeed,

$$
\theta_{1 j}=\min _{t \in[0,2 \pi]}\left[\arg z_{i}(t)-\tilde{q}_{j}(t)\right]-q_{0 j}, \quad \theta_{2 j}=\max _{t \in[0,2 \pi]}\left[\arg z_{i}(t)-\tilde{q}_{j}(t)\right]-q_{0 j} .
$$

So, all parameters of the circular domain of the first type $D_{\zeta}$ are found.

Now we have the functions $q_{s}(t), t \in[0,2 \pi], s=\overline{0, N}$, and, therefore, we can restore the relations between the boundary parameters of the domains $D_{z}$ and $D_{\zeta}$ via the formula $\theta_{s}(t)=\arg z_{s}(t)-q_{s}(t)$. Note that $\theta_{0}(t)$ grows monotonically when $t$ grows from 0 to $2 \pi, \theta_{0}(2 \pi)-\theta_{0}(0)=2 \pi$, while each of the functions $\theta_{s}(t), s=\overline{1, N}$, is $2 \pi$-periodic with one interval of increase and one interval of decrease. We can restore the inverse to $\theta_{0}(t)$, monotonically increasing the function $t_{0}(\theta)$, and we can restore the singlevalued functions $t_{s}^{ \pm}(\theta), \theta \in\left[\theta_{1 s}, \theta_{2 s}\right]$, where $t^{ \pm}$denotes the parameter of a twice traversed circular slit.

The approximate analytical function that maps $D_{\zeta}$ onto $D_{z}$ now has the form of the Cauchy integral

$$
f(\zeta)=\frac{1}{2 \pi} \int_{0}^{2 \pi} \frac{z_{0}\left(t_{0}(\theta)\right) e^{i \theta} d \theta}{e^{i \theta}-\zeta}+\sum_{s=1}^{N} \frac{1}{2 \pi} \int_{\theta_{1 s}}^{\theta_{2 s}} \frac{\left[z_{s}\left(t_{s}^{+}(\theta)\right)-z_{s}\left(t_{s}^{-}(\theta)\right)\right] R_{s} e^{i \theta}}{R_{s} e^{i \theta}-\zeta} d \theta .
$$

We can apply the Cauchy integral in the form

$$
f(\zeta)=\frac{1}{2 \pi} \int_{0}^{2 \pi} \frac{z_{0}(t) e^{i \theta_{0}(t)} \theta_{0}^{\prime}(t) d t}{e^{i \theta_{0}(t)}-\zeta}+\sum_{s=1}^{N} \frac{1}{2 \pi} \int_{0}^{2 \pi} \frac{z_{s}(t) R_{s} e^{i \theta_{s}(t)} \theta_{s}^{\prime}(t)}{R_{s} e^{i \theta_{s}(t)}-\zeta} d t
$$

in order not to deal with the functions $t_{s}^{ \pm}(\theta)$ and not to integrate along the different borders of the same slit.

It is worthy to apply the Laurent series expansions instead of the Cauchy integral for the representation of the function $z(\zeta)$. We have, for 
$\zeta \in D_{\zeta},|\zeta| \neq R_{s}, s=\overline{0, N}$, the series representation of the integrals in (5):

$$
\begin{aligned}
f(\zeta)= & \sum_{\substack{s=0 \\
|\zeta|<R_{s}}}^{N} \sum_{k=0}^{\infty} \frac{\zeta^{k}}{2 \pi R_{s}^{k}} \int_{0}^{2 \pi} z_{s}(t) e^{-i k \theta_{s}(t)} \theta_{s}^{\prime}(t) d t- \\
& -\sum_{\substack{s=1 \\
|\zeta|>R_{s}}}^{N} \sum_{k=0}^{\infty} \frac{R_{s}^{k}}{2 \pi \zeta^{k}} \int_{0}^{2 \pi} z_{s}(t) e^{i k \theta_{s}(t)} \theta_{s}^{\prime}(t) d t .
\end{aligned}
$$

We have, for $\zeta \in D_{\zeta}, \zeta=R_{j} e^{i \theta}, j \in\{1,2, \ldots, N\}, \theta \notin\left[\theta_{1 j}, \theta_{2 j}\right]$, the following representation

$$
\begin{aligned}
f\left(R_{j} e^{i \theta}\right)= & \frac{1}{4 \pi i} \int_{0}^{2 \pi} \frac{z_{j}(t) e^{i\left(\theta_{j}(t)-\theta\right) / 2} \theta_{j}^{\prime}(t) d t}{\sin \left(\left(\theta_{j}(t)-\theta\right) / 2\right)}+ \\
& +\sum_{\substack{s=0 \\
R_{j}<R_{s}}}^{N} \sum_{k=0}^{\infty} \frac{R_{j}^{k} e^{i k \theta}}{2 \pi R_{s}^{k}} \int_{0}^{2 \pi} z_{s}(t) e^{-i k \theta_{s}(t)} \theta_{s}^{\prime}(t) d t- \\
& -\sum_{\substack{s=1 \\
R_{j}>R_{s}}}^{N} \sum_{k=0}^{\infty} \frac{R_{s}^{k} e^{-i k \theta}}{2 \pi R_{j}^{k}} \int_{0}^{2 \pi} z_{s}(t) e^{i k \theta_{s}(t)} \theta_{s}^{\prime}(t) d t .
\end{aligned}
$$

Theorem 1 is proved.

Theorem 2. There exists an $(N+1)$-connected circular domain $D_{\zeta}$ of the second type and there exists a regular function $z=f(\zeta)$ in this domain such that the function $f(\zeta)$ maps conformally the domain $D_{\zeta}$ onto the given $(N+1)$-connected domain $D_{z}$ with smooth boundary components. The map is unique under the following conditions: the image of the inner circle $|\zeta|=r$ is the boundary component $L_{j}, j \in\{1,2, \ldots, N\}, f(1)=Z_{1}$, $Z_{1} \in L_{0}$. The corresponding approximate function can be constructed in the form of a Laurent polynomial.

Proof. We assume that $j=N$ and $\int_{0}^{2 \pi}\left(\arg z_{N}(t)\right)^{\prime} d t=-2 \pi$ without loss of generality. We construct the conformal map of the circular domain of the first type onto the domain $D_{z}$ by reparametrization of the given boundary 
representations. So, we search for functions $t_{s}(\theta), \theta \in[0,2 \pi], s=\overline{0, N}$, and for functions $t_{s}(\theta), \theta \in\left[\theta_{1 s}, \theta_{2 s}\right], s=\overline{1, N-1}$. The construction is as that for mapping of the circular domain of the first type.

We consider the analytic in the domain $D_{z}$ function $\zeta(z)$ that maps conformally the domain $D_{z}$ onto $D_{\zeta}$, and the analytic in $D_{z}$ function $\log \frac{z}{\zeta}$. We apply the boundary relation (1) as this has been done above. We introduce the functions $q_{s}(t)=\arg z_{s}(t)-\theta_{s}(t), s=\overline{0, N}$. After separation of the imaginary parts of the both sides of the previous equation, differentiation, and integration by parts, we get equation (2). We reduce the solution of the integral equation to the solution of a linear system with truncated matrices if we consider $q_{s}^{\prime}(t)$ representation (3). Now we have representation (4) for the functions $q_{s}(t)$. The constant summand $q_{00}$ can be restored in the same way as for the previous case. The values of $q_{0 s}$ and $R_{s}, s=\overline{1, N}$, can be also restored as has been done above with the help of the additional points $z_{s}^{*}, s=\overline{1, N}$, located in the exterior of the domain $D_{z}$. Note that $z_{N}^{*}=0$.

Finally, we have the mapping function in the series form

$$
\begin{aligned}
f(\zeta)= & \sum_{\substack{s=0 \\
|\zeta|<R_{s}}}^{N-1} \sum_{k=0}^{\infty} \frac{\zeta^{k}}{2 \pi R_{s}^{k}} \int_{0}^{2 \pi} z_{s}(t) e^{-i k \theta_{s}(t)} \theta_{s}^{\prime}(t) d t- \\
& -\sum_{\substack{s=1 \\
|\zeta|>R_{s}}}^{N} \sum_{k=0}^{\infty} \frac{R_{s}^{k}}{2 \pi \zeta^{k}} \int_{0}^{2 \pi} z_{s}(t) e^{i k \theta_{s}(t)} \theta_{s}^{\prime}(t) d t
\end{aligned}
$$

for $\zeta \in D_{\zeta},|\zeta| \neq R_{s}, s=\overline{0, N}$.

We offer a new method of finding the values of $q_{0 s}, R_{s}, s=\overline{1, N}$, in order to avoid using many additional points $z_{j}^{*}, j=\overline{1, N-1}$, and to deal only with the point $z_{N}^{*}=0$. Let $\tilde{\theta}_{s}(t)=\arg z_{s}(t)-\tilde{q}_{s}(t)$. Now we have the following integral representation of the mapping function for $\zeta \in D_{\zeta}$ :

$$
f(\zeta)=\frac{1}{2 \pi} \int_{0}^{2 \pi} \frac{z_{0}(t) e^{i \theta_{0}(t)} \theta_{0}^{\prime}(t) d t}{e^{i \theta_{0}(t)}-\zeta}+\sum_{s=1}^{N} \frac{1}{2 \pi} \int_{0}^{2 \pi} \frac{z_{s}(t) Q_{s} e^{i \tilde{\theta}_{s}(t)} \tilde{\theta}_{s}^{\prime}(t)}{Q_{s} e^{i \tilde{\theta}_{s}(t)}-\zeta} d t
$$

where $Q_{s}=R_{s} e^{-i q_{0 s}}, s=\overline{1, N}$ are unknown. The Cauchy integral vanishes at the points outside $D_{\zeta}$. So, the expression at the right-hand side of (6) 
and all derivatives of this expression vanish at the point $\zeta=0$. Therefore, we have for all $j \in \mathbf{N}$

$$
\int_{0}^{2 \pi} z_{0}(t) e^{i(1-j) \theta_{0}(t)} \theta_{0}^{\prime}(t) d t+\sum_{s=1}^{N} \int_{0}^{2 \pi} z_{s}(t)\left(Q_{s} e^{i \tilde{\theta}_{s}(t)}\right)^{(1-j)} \tilde{\theta}_{s}^{\prime}(t) d t=0 .
$$

We apply relations (7) in order to find the values of $Q_{s}=R_{s} e^{-i q_{0 s}}$, $s=\overline{1, N}$, and, therefore, the values of $q_{0 s}, R_{s}, s=\overline{1, N}$. The least-squares method can be applied. After we find these values, we apply formula (6) as the representation of the mapping function.

4. Examples of conformal mappings. In the first example, we apply the conformal mapping from [10]; the parametrization of the boundaries is as follows:

$$
\begin{aligned}
& L_{0}=\left\{z=4+6 i+(10+3 \cos 3 t) e^{i t}\right\}, \\
& L_{1}=\left\{z=0.5+12 i+0.5 e^{-i \pi / 4}\left(e^{i t}+4 e^{-i t}\right)\right\}, \\
& L_{2}=\left\{z=9+6 i+0.5 e^{i \pi / 4}\left(e^{i t}+4 e^{-i t}\right)\right\}, \\
& L_{3}=\left\{z=0.5+0.5 e^{i \pi / 4}\left(e^{i t}+4 e^{-i t}\right)\right\} .
\end{aligned}
$$

Recall that [10] describes construction of the conformal mapping of a bounded multiply connected region onto an annulus with circular slits and we are solving the inverse problem: we construct an approximate analytical conformal mapping of an annulus with circular slits onto the given multiply connected domain.

The result of the conformal mapping is shown in Figure 1.
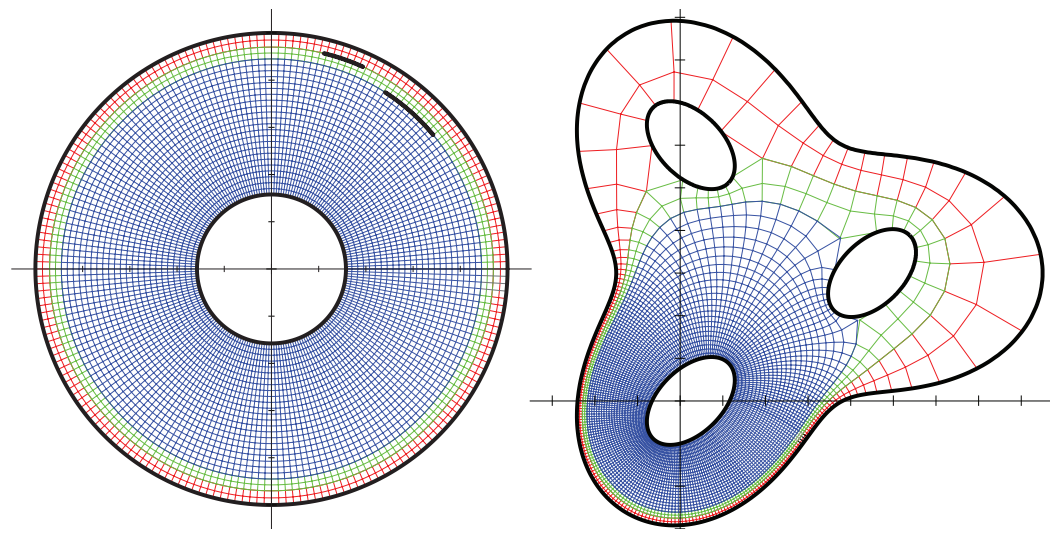

Figure 1: Domains $D_{\zeta}$ and $D_{z}$ in example 1. 
We also investigated the effect of the number $M$ of the Fourier coefficients on the parameters of the problem and accuracy.

Table 1: Accuracy and the elapsed time for example 1.

\begin{tabular}{|c|c|c|c|c|c|}
\hline$M$ & $R_{1}$ & $R_{2}$ & $R_{3}$ & $\varepsilon$ & Time $(\mathrm{s})$ \\
\hline 25 & 0.939091 & 0.888035 & 0.315839 & $0.4916 \cdot 10^{-3}$ & 0.28 \\
50 & 0.939829 & 0.889457 & 0.315620 & $0.3010 \cdot 10^{-4}$ & 0.42 \\
100 & 0.940017 & 0.889814 & 0.315564 & $0.1862 \cdot 10^{-5}$ & 1.07 \\
200 & 0.940063 & 0.889902 & 0.315550 & $0.1158 \cdot 10^{-6}$ & 4.07 \\
400 & 0.940074 & 0.889924 & 0.315546 & $0.7219 \cdot 10^{-8}$ & 19.84 \\
\hline
\end{tabular}

Table 1 presents the results; it also shows the elapsed time. Calculations were carried out on a computer Intel Core i5-3330 3Ghz CPU, 4Gb RAM, all the computations were done using Fortran (double precision).
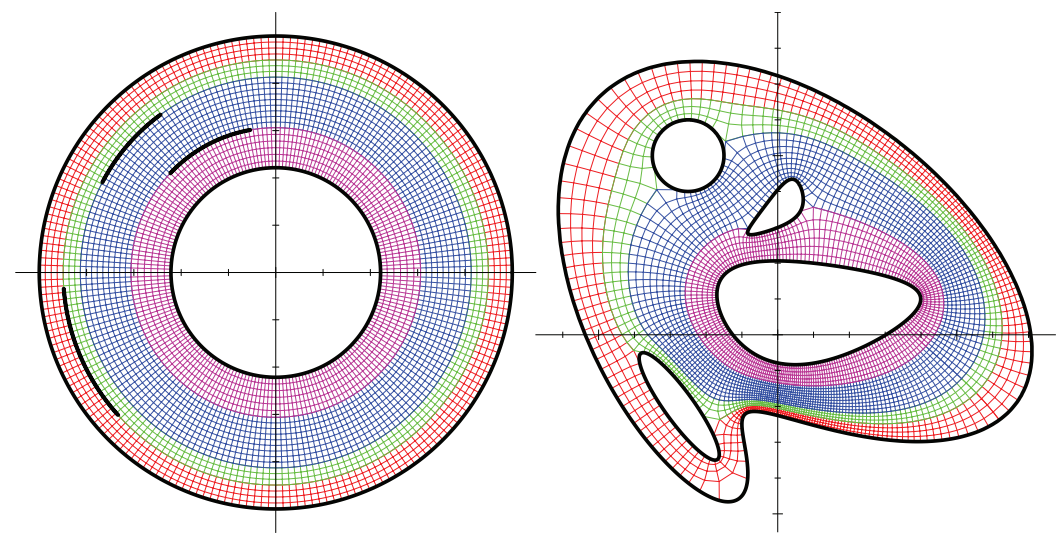

Figure 2: Domains $D_{\zeta}$ and $D_{z}$ in example 2 .

Figure 2 shows an example of a conformal mapping on the domain with non-convex and non-starlike boundary. The boundary parametrization is

$$
\begin{aligned}
& L_{0}=\left\{z=e^{i t}-0.5 i e^{2 i t}+0.2 e^{-2 i t}\right\}, \\
& L_{1}=\left\{z=-0.55-0.4 i-0.1(1-i) e^{i t}-0.1(1-2 i) e^{-i t}\right\}, \\
& L_{2}=\left\{z=-0.5+i+0.2 e^{-i t}\right\}, \\
& L_{3}=\left\{z=0.7 i+(1+i)\left(0.04 e^{i t}+0.1 e^{-i t}\right)-0.02 e^{2 i t}\right\}, \\
& L_{4}=\left\{z=0.2+0.15 i-0.1(1+i) e^{i t}-0.3(1-i) e^{-i t}-0.03(1-i) e^{2 i t}\right\} .
\end{aligned}
$$

In the following example, we consider the case of $0 \in D_{z}$. The domain 
$D_{z}$ is the disk with four cut out smaller disks:

$$
\begin{array}{r}
L_{0}=\left\{z=4 e^{i t}\right\}, \quad L_{1}=\left\{z=2+e^{-i t}\right\}, \quad L_{2}=\left\{z=-2+e^{-i t}\right\}, \\
L_{3}=\left\{z=2 i+e^{-i t}\right\}, \quad L_{4}=\left\{z=-2 i+e^{-i t}\right\} .
\end{array}
$$

An example of a conformal mapping onto the domain is shown by Figure 3.

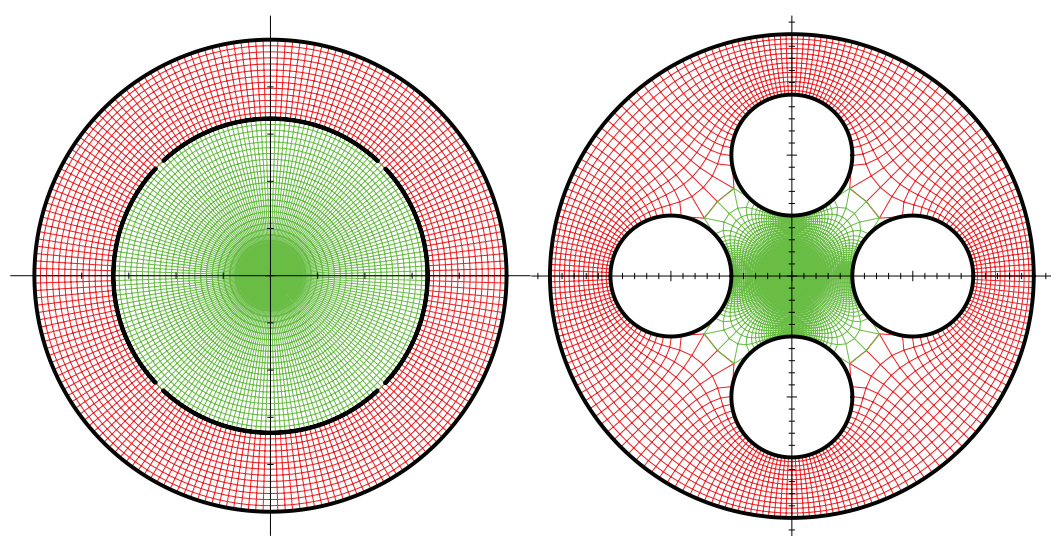

Figure 3: Domains $D_{\zeta}$ and $D_{z}$ in example 3.

5. Conclusion. It is possible to construct the approximate conformal mapping of the unit disk or an annulus with circular slits onto the given multiply connected domain by Laurent polynomial functions in each annular region. The solution can be reduced to a finite linear system. The method is easily computable.

\section{References}

[1] Schinzinger R., Laura P. A. A. Conformal Mapping: Methods and Applications. Dover Publications, 2003.

[2] Crowdy D., Marshall J. Conformal Mappings between Canonical Multiply Connected Domains. Computational Methods and Function Theory, 2006, vol. 6, pp. 59-76. DOI: https://doi.org/10.1007/ $\mathrm{BF} 03321118$

[3] Murid A. H. M., Laey-Nee Hu. Numerical conformal mapping of bounded multiply connected regions by an integral equation method. Int. J. Contemp. Math. Sci., 2009, vol. 4, pp. 1121-1147.

[4] Wegmann R. Fast conformal mapping of multiply connected regions. J. Comput. Appl. Math., 2001, vol. 130, pp. 119-138. DOI: https: //doi.org/10.1016/S0377-0427(99)00387-8. 
[5] Wegmann R., Nasser M. M. S. The Riemann-Hilbert problem and the generalized Neumann kernel on multiply connected regions. J. Comput. Appl. Math., 2008, vol. 214, pp. 36-57. DOI: https: //doi.org/10.1016/j.cam.2007.01.021.

[6] Wen G C. Conformal mapping and boundary value problems (English transl. of Chinese edition 1984). Providence, RI: American Math. Soc., 1992.

[7] Nasser M. M. S. A boundary integral equation for conformal mapping of bounded multiply connected regions. Comput. Methods Funct. Theory, 2009, vol. 9, pp. 127 -143. DOI: https://doi.org/10.1007/ BF03321718.

[8] Nasser M. M. S., Al-Shihri F. A. A. A fast boundary integral equation method for conformal mapping of multiply connected regions. SIAM J. on Sci. Comp., 2013, vol. 35(3) pp. 1736-1760. DOI: https:// doi.org/10.1137/120901933.

[9] Sangawi A. W. K., Murid A. H. M., Nasser M. M. S. Linear integral equations for conformal mapping of bounded multiply connected regions onto a disk with circular slits. Appl. Math. Comput., 2011, vol. 218, pp. 2055-2068. DOI: https://doi.org/10.1016/j.amc. 2011.07 .018$.

[10] Sangawi A. W. K., Murid A. H. M., Nasser M. M. S. Annulus with circular slit map of bounded multiply connected regions via integral equation method. Bull. Malays. Math. Sci. Soc., 2012, vol. 2(35), pp. $945-969$.

[11] Yunus A. A. M., Murid A. H. M., Nasser M. M. S. Numerical conformal mapping and its inverse of unbounded multiply connected regions onto logarithmic spiral slit regions and straight slit regions Proc. of the Royal Society A - Math. Phys. and Eng. Sci., 2014, vol. 470(2162), Article No. 20130514. DOI: https://doi.org/10.1098/rspa. 2013. 0514.

[12] Yunus A. A. M., Murid A. H. M., Nasser M. M. S. Numerical evaluation of conformal mapping and its inverse for unbounded multiply connected regions. Bull. Malays. Math. Sci. Soc., 2014, vol. 1(24), pp. $1-24$.

[13] Wegmann R. Methods for numerical conformal mappings. In Handbook of complex analysis: geometric function theory, vol. 2, Amsterdam, The Netherlands: Elsevier, 2005, pp. 351-477. 
[14] Shirokova E. A. On the approximate conformal mapping of the unit disk on a simply connected domain. Russian Math., 2014, vol. 58, pp. 47 -56. DOI: https://doi.org/10.3103/S1066369X14030050.

[15] Shirokova E. A., Ivanshin P. N. Approximate Conformal Mappings and Elasticity Theory. J. of Compl. Analysis, 2016. DOI: https:// doi .org/10.1155/2016/4367205.

[16] Abzalilov D. F., Shirokova E. A. The approximate conformal mapping onto simply and doubly connected domains. Complex Variables and Elliptic Equations, 2017, vol. 62, pp. 554-565. DOI: https://doi . org/10.1080/17476933.2016.1227978.

[17] DeLillo T. K. The Accuracy of Numerical Conformal Mapping Methods: A Survey of Examples and Results. SIAM J. Num. Anal., 1994, vol. 31. DOI: https://doi.org/10.1137/0731043.

[18] DeLillo T. K. On some relations among numerical conformal mapping methods. J. Comput. Appl. Math., 1987, vol. 19, pp. 363-377. DOI: https://doi .org/10.1016/0377-0427(87)90205-6.

[19] Henrici P. Applied and computational complex analysis, New York, NY: John Wiley. 1986, vol. 3.

[20] Gakhov F. D. Boundary value problems. Pergamon Press, Oxford, 1966.

Received July 30, 2018.

In revised form, December 19, 2018.

Accepted December 20, 2018.

Published online January 5, 2019.

Kazan Federal University

18 Kremlyovskaya str Kazan, 420008, Russia

E-mail:

D. F. Abzalilov

damir.abzalilov@kpfu.ru,

E. A. Shirokova

elena.shirokova@kpfu.ru 